\title{
Correction to: Newly Introduced or Modified Genes in Plants Potentially Modulate the Host Microbiome
}

Feth el Zahar Haichar and Wafa Achouak

\section{Correction to: \\ Chapter 9 in: V. V. S. R. Gupta, A. K. Sharma (eds.), Rhizosphere Biology: Interactions Between Microbes and Plants, Rhizosphere Biology, https://doi.org/10.1007/978-981-15-6125-2_9}

The original version of the chapter was inadvertently published with an error in author affiliation. Author Feth el Zahar Haichar is also affiliated to "INSA-Lyon, Université Claude Bernard Lyon1, CNRS, UMR5240, Microbiologie, Adaptation, Pathogénie, Univ Lyon, 10 rue Raphaël Dubois, 69622, Villeurbanne, France”. The chapter and list of contributors in FM has now been corrected by including the second affiliation. 